\title{
Reverse engineering and CAD-CAM approach for manufacturing of spare parts. Case study
}

\author{
Iulian Stănăşel ${ }^{1, *}$, Florin Blaga $^{1}$, Traian Buidoş ${ }^{1}$ and Dan Crăciun $^{2}$ \\ ${ }^{1}$ University of Oradea, Faculty of Management and Technological Engineering, Industrial Engineering Department, Romania \\ ${ }^{2}$ University of Oradea, Faculty of Management and Technological Engineering, Mechatronics Department, Romania
}

\begin{abstract}
Reverse engineering represents a method to retrieve information from an existing product, information which are then used for designing a new product or to reproduce it in order to make spare parts. In this paper is presented a case study on making a spare part of complex shape from a worn part. There are presented the stages of acquisition of data by scanning, the use of reverse engineering for the realization of the $3 \mathrm{D}$ model, the virtual manufacturing and then the machining of the workpiece $\mathrm{CNC}$ machine tools.
\end{abstract}

\section{Introduction}

In the present time due to factors such as increased competition, the market pressure, the development cycle of new products tends to become shorter. The process of achieving a product consists of several stages Fig. 1.

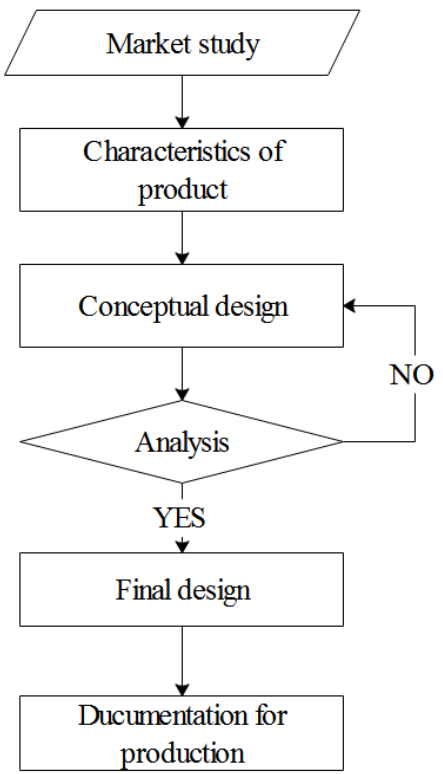

Fig. 1. The main steps of reverse engineering process.

The amount of information in the field of engineering knows an unprecedented development which is why software applications have become increasingly more complex and faster, allowing the integration of activities of design, analysis, and manufacturing of the virtual prototype.

New software technologies make possible to design and study the behavior of a new product in the virtual environment with high accuracy, which has a high impact on reducing the development cycle.
In accordance by [1], Reverse engineering is a process by which digital data are obtained in the form of a cloud of points belonging to the surfaces of a physical object, which are processed and transformed into a 3D model of the scanned object.

Digital data acquisition can be done by measuring or 3D scanning. Although numerically controlled machines are more accurate than the 3D scanners, they offer the advantage of having higher productivity under right precision conditions [2].

The large amounts of data needed to be processed make it necessary to use high-performance computers equipped with dedicated applications with the aim to increase the working speed and to achieve a high quality of the 3D model.

Reverse engineering is used for various purposes [3]:

- $\quad$ for the reproduction of a piece (as a spare part) in the situation where there is no longer any documentation of execution, when the producer no longer exists, or the workpiece is no longer produced, but there are requests from the customers.

- analyzing some features and exploring possibilities to enhance the performance of an existing product.

- to create a new product with improved performances, by redesigning an existing product

- $\quad$ to achieve the 3D model of a product based on a physical model made by artists in clay, wood or plaster.

- $\quad$ to create3D models of objects from nature or to reproduce on a specific scale in order to produce them.

- for protheses design and manufacturing in the medical field

- $\quad$ in archeology to rebuild missing parts of certain objects by using data from existing parts

\footnotetext{
* Corresponding author: stanasel@uoradea.ro
} 


\section{Obtaining the 3D model through reverse engineering}

Obtaining the 3D model of the object which must be carried out, involves the following steps ( Fig. 2.)

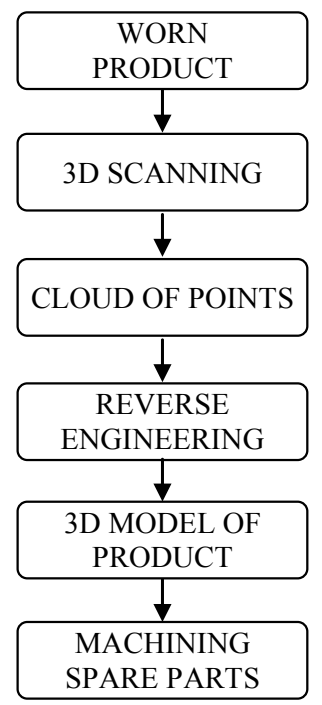

Fig. 2. The main steps of reverse engineering process.

For scanning the physical object it was used a numerically controlled rotary table that can be programmed for different angles, and a 3D scanner Optim II supplied with blue light and two video cameras.

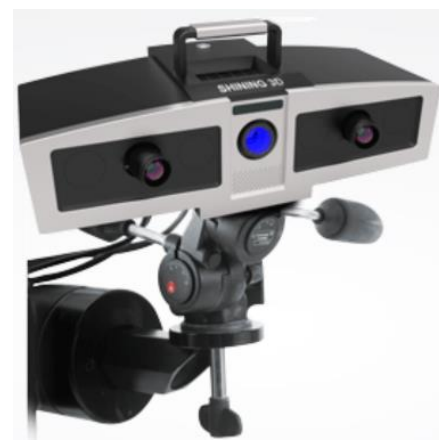

Fig. 3. The 3D scanner

The physical object that had to be reproduced is shown in Fig. 4. From its analysis, it can be seen that it is delimited by several curved surfaces. For this reason the determination of dimensions by measuring with traditional instruments is practically impossible.

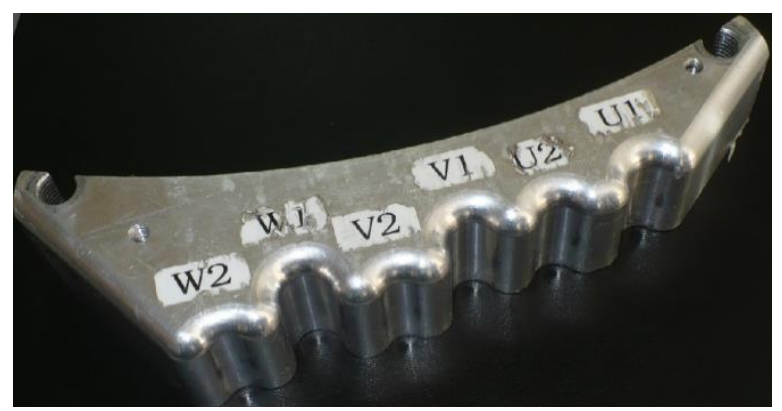

Fig. 4. The worn part.
On the other reason, the accuracy required to the achieved workpiece is within \pm 0.1 , which is ensured by the used scanner.

They have performed ten scans at equidistant angles until a complete rotation of the workpiece. The data resulting from scanning are shown in Fig. 5. These were saved, and for their processing, it was used reverse engineering application Shiningform XOR.

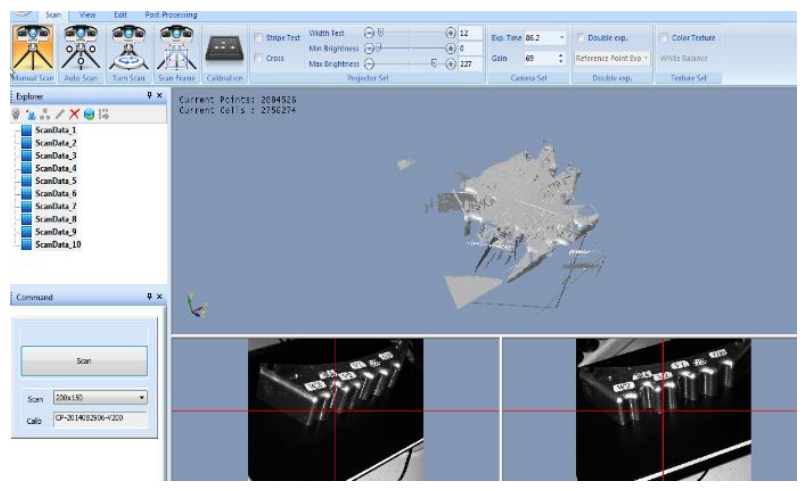

Fig. 5. The unaligned scanned data

For the construction of the workpiece surfaces, the points resulting from the scan are subjected to several operations performed in several stages. In the first phase, each set of data corresponding to the successive scans was cleaned by the parasitic points which come from the objects located in the visual field of the two cameras of the $3 \mathrm{D}$ scanner.

Then was done the alignment of the data for each scan, the operation of which depends on the accuracy with which the scanned object was reproduced. The application allows you to determine the accuracy of the cloud of points alignment by displaying a coloured map, in which green colour corresponds to surface that is in interval $\pm 0.1 \mathrm{~mm}$ which is prescribed by the beneficiary of the parts (Fig. 6).
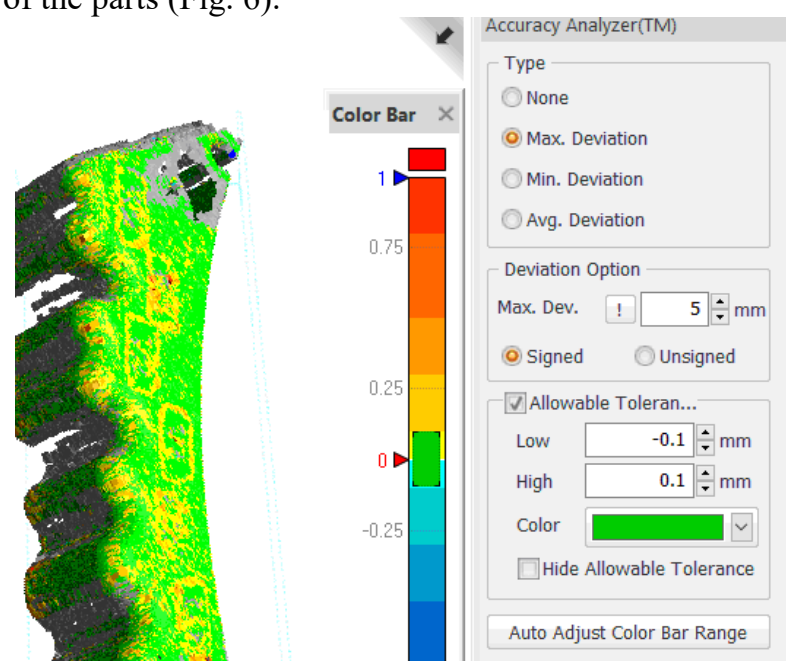

Fig. 6. Accuracy analyser.

The cloud of points of the scanned object is shown in Fig. 7. Because the beneficiary did not allow the application of a layer of matte paint on the surface of the piece, the mesh shows some discontinuities due to the 
metallic gloss. However, the surface shows enough points which allow determining the correct shape of the object.

The cloud of points is exported to the Siemens PLM $\mathrm{NX}$ application, which allows using the scanned data to create the sketch needed to extrude the 3D model of the object.

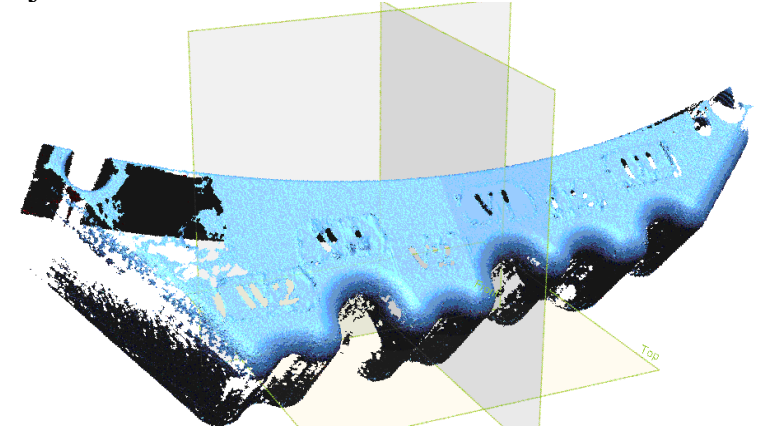

Fig. 7. The cloud of points of the scanned object.

In order to obtain the sketch required to extrude the solid model, the mesh is intersected with several parallel planes to get as many points on the contour in order to represent correctly the surfaces with discontinuities. (Fig. 8).

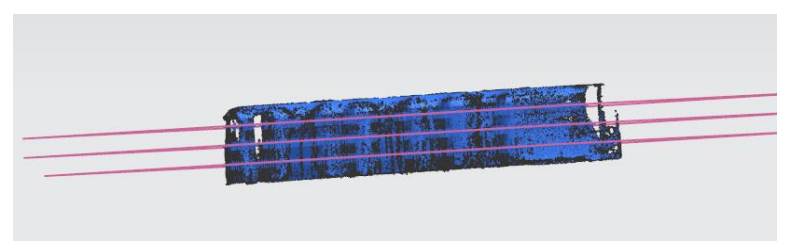

Fig. 8. Intersections with parallel planes

The points obtained were projected onto the plane on which the sketch is built and were joined by using specific commands to define the contour of the workpiece (Fig. 9).

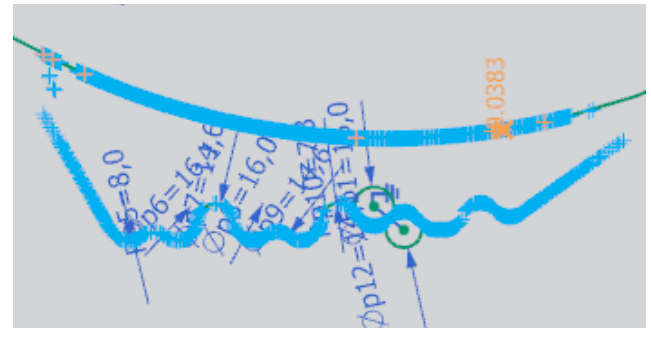

Fig. 9. The sketch of the part

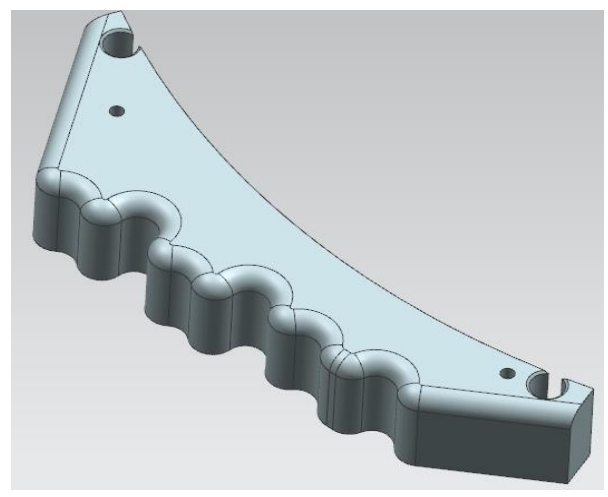

Fig. 10. The 3D model of the scanned object
By extruding the outline and applying chamfering and filleting operations, they obtained the 3D model [4] of the scanned object (Fig. 10).

\section{Manufacturing of the spare part}

Taking into account the complexity of the workpart, it is proposed to be done on numerically controlled machine tools. There were determined the machining operations, the needed cutting tools, and were calculated the cutting parameters.

The cutting parameters were determined by using an application which contains a database of the different materials and cutting tools. In Fig. 11 there are shown the parameters of the drilling operation.

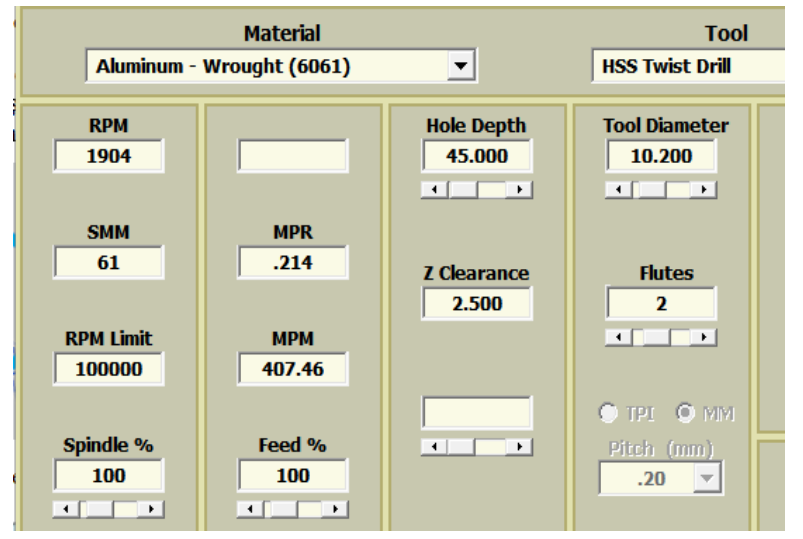

Fig. 11. The geometrical parameters of the drilling tool.

Table 1 shows the machining operations, the cutting parameters and the cutting times calculated for each operation.

Table 1. The necessary operations for manufacturing the part.

\begin{tabular}{|l|c|c|c|c|}
\hline \multicolumn{1}{c}{ Operation } & $\begin{array}{c}\text { Tool } \\
\text { Number }\end{array}$ & Speed & Feed & Time \\
\cline { 2 - 5 } & & rot/min & $\mathrm{mm} / \mathrm{min}$ & $\mathrm{min}$ \\
\hline Spot_drilling & 1 & 1618 & 196 & $00: 00: 17$ \\
\hline Deep_hole_drilling_d4.8 & 2 & 4000 & 450 & $00: 00: 20$ \\
\hline Deep_hole_drilling_d10.2 & 3 & 1900 & 400 & $00: 00: 16$ \\
\hline Countersinking & 4 & 950 & 300 & $00: 00: 03$ \\
\hline Reaming_d5 & 5 & 2900 & 300 & $00: 00: 20$ \\
\hline Tapping_m12 & 6 & 800 & 300 & $00: 00: 20$ \\
\hline Milling_contour & 7 & 4000 & 1200 & $00: 11: 34$ \\
\hline Fillet_mill & 8 & 4000 & 1000 & $00: 07: 25$ \\
\hline & & & & $00: 22: 13$ \\
\hline
\end{tabular}

To determine the paths for each tool used to make the workpiece, it was used a Computer Aided Manufacturing (CAM) application [5].

Using the application for digital production involves going through several stages. It was established the origin of the coordinate system for manufacturing, there were indicated the geometry of the blank and the geometry of the workpiece. For each operation, were defined the cutting tool, the surfaces to be machined, cutting parameters, cutting strategy. 
As an example, in Fig. 12 are shown the geometrical parameters for the drill Ø10.2 used to make the threaded hole.

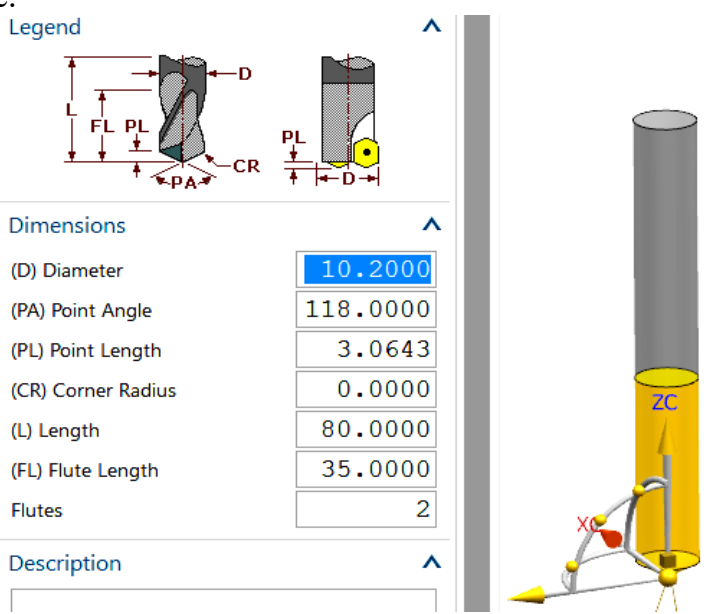

Fig. 12. The geometrical parameters of drilling tool.

All the tool paths for manufacturing the workpiece are shown in Fig. 13.

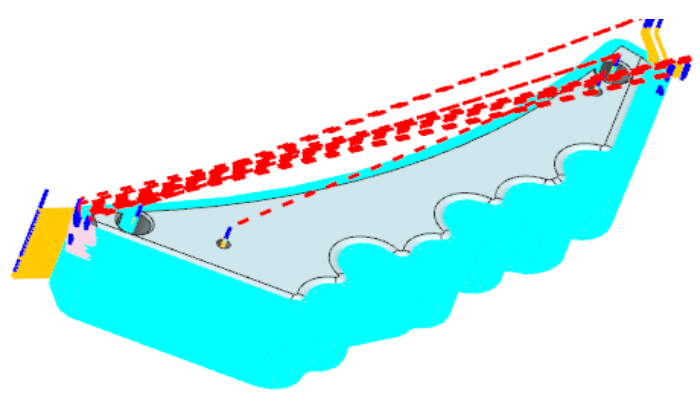

Fig. 13. The tools paths for manufacturing

After generating the tools paths, it is possible to check them by $3 \mathrm{D}$ simulation. By measuring of the resultant piece after virtual manufacturing, it is possible to highlight collisions or to identify the areas which are inappropriately processed (Fig. 14).

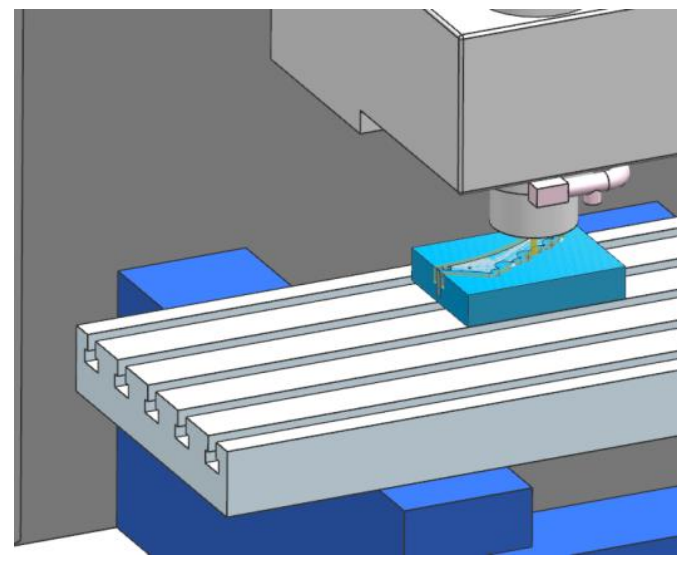

Fig. 14. The 3D simulation of the tools paths.

The postprocessing was done for a HAAS VF3 computer numerically controlled (CNC) equipment on which the workpiece was machined. The running of the program on the CNC machine tool is shown in Fig. 15, and in Fig. 16 it is shown the workpiece obtained after machining.

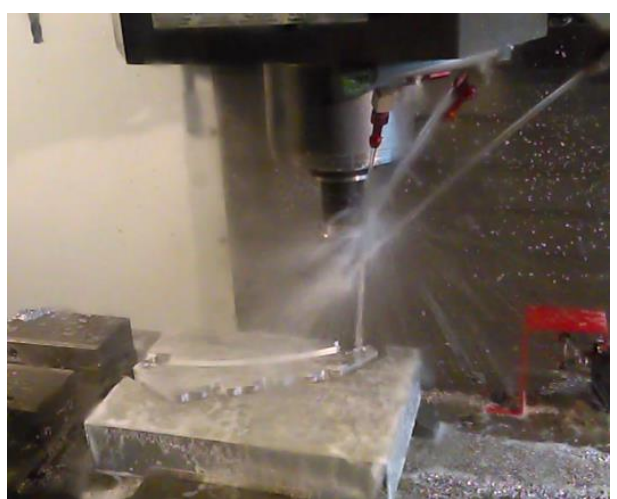

Fig. 15. Aspect during the processing of the piece

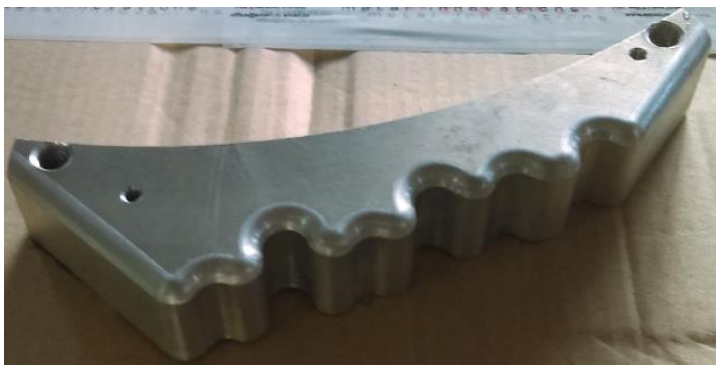

Fig. 16. The machined part

\section{Conclusions}

The case study shows the use of reverse engineering and CAM techniques to obtain a spare part of complex shape for which there is no documentation nor the manufacturer is known, starting from a worn part.

The digital data obtained through 3D scanning was used to get the 3D model of the part.

It was done the virtual machining of the3D model using a CAM application.

The CNC program obtained after post-processing was tested and back plotted before running on the HAAS VF2 equipment.

The work is a part of project MANUNET ERA-NET, Contract nr.19/2015 "Monorail conveyor system for automated production lines".

\section{References}

1. R. Abella, J. Daschbach, R. McNichols Reverse engineering applications. Comput Ind Eng, (1994).

2. D. Nedelcu, R. Avasiloaie, D. Florea, A. Rajic, Applications of the Rapid Prototyping Technology to Manufacture the Pelton Runners, Anale Univ. "Eftimie Murgu" Reşiţa, anul XX, 1, (2013).

3. W. Wego, Reverse Engineering: Technology of Reinvention, CRC Press, Taylor \& Francis, (2010).

4 G. Manole, E. Oprea, M. Iosip, Concept and design of products (Conceptia si proiectarea produselor), Qual Media, (2009).

5. M. Iosip, E. Oprea, D. Boricean, Performing digital manufacturing of products unsing virtual prototyping (Realizarea fabricatiei digitale a produselor folosind prototipul virtua)l, Qual Media, (2010) 